\title{
1D Infrared Camera Distortion Based on Polynomial Model
}

\author{
Qin Wenjian, Li Zhicheng, Gu Jia \\ Research Centre for Medical Robotics and Minimally \\ Invasive Surgical Devices, \\ Shenzhen Institutes of Advanced Technology, \\ Chinese Academy of Sciences \\ Shenzhen, China, 518055
}

\author{
Chen Ke ,Zhang Xiaoping \\ Lenovo Corporate R\&D, \\ Beijing, China, 100085
}

\begin{abstract}
In this paper, we propose a novel method for 1D infrared camera distortion based on polynomial model. We derive a 1D camera calibration algorithm and then estimate the distortion parameters by means of minimum calibration error. The used polynomial model is linear, such that the estimation can be finished with low computation burden without losing accuracy. The proposed method is validated with real images. The experimental results show that the distortion parameters can be estimated with high accuracy up to about 2-pixel mean error in less than 10 iterations.
\end{abstract}

Keywords-Camera calibration; Lens distortion; Infrared camera

\section{INTRODUCTION}

Infrared ray imaging has been widely used in many fields, such as infrared spectrum for disease diagnosis [1], industry examined [2], astronomy [3]. Infrared camera [4] has been quickly developed with development of electronic technology, which has advantages of low cost and high quality. In our study, we use infrared camera for real computer screen image coordinate, and then we reconstructed the world coordinate of computer screen by projective algorithm. However, due to limitation of the manufacturer technology, lens distortion can not be avoided, which has a great negative influence to the quality of imaging. Until recently, The lens with high imaging quality has been tested under awfully rigid condition. Unfortunately, different degrees of distortion has still been produced, which results in much error for image processing, especially for camera calibration. Although this problem can not be eliminated, we are able to reduce the distortion degree by use of hardware or software improvement.

The problem of camera distortion has attracted the attention of researchers in the computer vision community, and many papers have been devoted to seek the optimal mathematics distortion model for different lens or different application environment. The distortion included radial and tangent [5], while the tangent distortion can be ignored in the practical application. So the radial distortion is a significant problem in the analysis of digital images. R.I.Hartley and A. Zisserman [6] developed the polynomial model for wide angel lens. A. W. Fitzgibbon [7] showed that for low distortion, it is possible to obtain a solution for both epipolar geometry and a single parameter of lens distortion. F. Devernay and O. Faugeras [8] proposed a model based on the fish-eye lens design parameters, this physical basis results in an excellent distortion fit. The rational function model [14] for wide-angel and catadioptric lenses is derivate of the rational cubic camera described by R. I. Hartley and T. Saxena [9], which is not specialized to any particular lens geometry.

The core of lens distortion problem is how to solve distortion parameters incorporated the algebra method or geometry constraints. There are various distortion models for camera calibration, but there is no general algorithm for any lens since we are not clear that the really causing. The polynomial model has been widely used in various field, which is simple and easy to implement, resulting in fast computation. The method [6] is also most popular in computer vision for distortion, choosing different orders according to requirement of accuracy. In additional, currently most of distortion methods is derived from 2D image information or geometry constraints [10]. However, there are few papers addressing the problem of 1D camera calibration and distortion.

In this paper, we propose a novel method for 1D infrared camera distortion based on polynomial model. We derive the 1D camera calibration algorithm and then employ the minimum calibration error for distortion parameters solution. The used polynomial model is linear, such that the estimation can be finished with low computation burden without losing accuracy. Our method is generic enough because it allows for any algebraic distortion model without making any extra assumption of the distortion model.

\section{Description Of The Proposed Algorithm}

Currently, a lot of researchers have devoted to lens distortion, and developed many algorithms. But the drawback of the previous works [6-11] depended on 2D image information, such as straight line or epipolar geometry constraints. Nevertheless, in our study, we have to solve 1D camera distortion problem. The points in 1D space are independent, among which have no relationship. Unlike the information of straight line in the $2 \mathrm{D}$ images. So it is bound to the difficulty of utilizing the presently method to realize undistortion. In this paper, we proposed a novel method for 1D infrared camera distortion after analyzing the cause of infrared camera distortion. The proposed method is a derivate of the polynomial model, and here we reduce the polynomial with minimum calibration error. We can obtain a 
exactness, robust and reliable distortion parameters for 1D camera undistortion.

\section{A. Proposed $1 D$ camera calibration algorithm}

This section explains the theory of calibration that is important for distortion, we briefly sketch this method. The camera imaging model is originated from pin-hole model [6], we reduce the model to find the map between 1D image coordinate and 2D world coordinate. Let $\mathrm{u}$ and [xw, yw] respectively denote image space coordinate and world screen coordinate. We can get projective map as :

$$
y_{c}\left[\begin{array}{l}
u \\
1
\end{array}\right]=\boldsymbol{M}\left[\begin{array}{c}
x_{w} \\
y_{w} \\
1
\end{array}\right]=\left[\begin{array}{lll}
m_{11} & m_{12} & m_{13} \\
m_{21} & m_{22} & m_{23}
\end{array}\right]\left[\begin{array}{c}
x_{w} \\
y_{w} \\
1
\end{array}\right]
$$

Where $\mathrm{yc}$ is scale factor, and the $2 \times 3$ projective

$$
\mathbf{M}=\left[\begin{array}{lll}
m_{11} & m_{12} & m_{13} \\
m_{21} & m_{22} & m_{23}
\end{array}\right] \text { is the calibration matrix, which }
$$

we need to estimate. After calibration, we can get the projective map between image space and screen space, and thus the [xw, yw] can be computed accurately.

The (1) is also written as:

$$
m_{11} x_{w}+m_{12} y_{w}-m_{21} x_{w} u-m_{22} y_{w} u+m_{13}-m_{23} u=0
$$

There are correspondence (3) for every sample point

$m_{11} x_{w i}+m_{12} y_{w i}-m_{21} x_{w i} u_{i}-m_{22} y_{w i} u_{i}+m_{13}-m_{23} u_{i}=0$

There are six unknown parameters in projective matrix, so we need $n \geq 6$ sample points and we can get $n$ equations about $m_{i j}$, the expression is given:

$$
\left[\begin{array}{ccccc}
x_{w 1} & y_{w 1} & -x_{w 1} u_{1} & -y_{w 1} u_{1} & 1 \\
\vdots & \vdots & \vdots & \vdots & \vdots \\
x_{w n} & y_{w n} & -x_{w n} u_{n} & -y_{w n} u_{n} & 1
\end{array}\right]\left[\begin{array}{l}
m_{11} \\
m_{12} \\
m_{21} \\
m_{22} \\
m_{13}
\end{array}\right]=m_{23}\left[\begin{array}{c}
u_{1} \\
\vdots \\
u_{n}
\end{array}\right]
$$

Usually, let $m_{23}=1$, so the(4) is represented as linear equation $\mathbf{A L}=\mathbf{U}$, and we know that the six unknown elements in projective matrix are dependent [11], which is related to two intrinsic parameters and four extrinsic parameters included two rotations and two translations. We can make use of the constraints above mentioned to solve projective matrix. For camera intrinsic parameters are constant, we can derive that Frobenius norm of projective matrix is constant. As result, we can solve (4) by the constraints above mentioned.

\section{B. Proposed distortion algorithm}

Polynomial model is the most popular model to describe radial distortion. We expand the distortion model with six orders Taylor series, and we can get:

$$
u^{\prime}=k_{0}+k_{1} u+k_{2} u^{2}+k_{3} u^{3}+k_{4} u^{4}+k_{5} u^{5}+k_{6} u^{6}
$$

Where $\boldsymbol{K}=\left[\begin{array}{lllllll}k_{0} & k_{1} & k_{2} & k_{3} & k_{4} & k_{5} & k_{6}\end{array}\right]^{T}$ is distortion parameters, and $\mathrm{u}$ is the original image coordinate of touch points, $u^{\prime}$ is the undistortion coordinate.
We can get distortion parameters by use of calibration points after calibration, the (2) can be rewritten with using the $u$ instead of $u^{\prime}$ :

$$
\begin{aligned}
& \left(m_{21} x_{w}+m_{22} y_{w}+m_{23}\right) u^{\prime}=m_{11} x_{w}+m_{12} y_{w}+m_{13} \\
& \text { e.g } E=m_{21} x_{w}+m_{22} y_{w}+m_{23}, F=m_{11} x_{w}+m_{12} y_{w}+m_{13},
\end{aligned}
$$
using (7) in (5) we get:

$$
E\left(k_{0}+k_{1} u+k_{2} u^{2}+k_{3} u^{3}+k_{4} u^{4}+k_{5} u^{5}+k_{6} u^{6}\right)=F
$$

Put whole calibration points into (7), so get

$$
\begin{gathered}
\left(\begin{array}{ccccc}
E_{0} & E_{0} u_{0} & E_{0} u_{0}{ }^{2} & \cdots & E_{0} u_{0}{ }^{6} \\
\vdots & \vdots & \vdots & \vdots & \vdots \\
E_{n} & E_{n} u_{n} & E_{n} u_{n}{ }^{2} & \cdots & E_{n} u_{n}{ }^{6}
\end{array}\right)\left(\begin{array}{c}
k_{0} \\
k_{1} \\
k_{2} \\
k_{3} \\
k_{4} \\
k_{5} \\
k_{6}
\end{array}\right)=\left(\begin{array}{c}
F_{0} \\
F_{1} \\
F_{2} \\
F_{3} \\
F_{4} \\
F_{5} \\
F_{6}
\end{array}\right) \\
\text { Let } \boldsymbol{E}=\left(\begin{array}{ccccc}
E_{0} & E_{0} u_{0} & E_{0} u_{0}{ }^{2} & \cdots & E_{0} u_{0}{ }^{6} \\
\vdots & \vdots & \vdots & \vdots & \vdots \\
E_{n} & E_{n} u_{n} & E_{n} u_{n}{ }^{2} & \cdots & E_{n} u_{n}{ }^{6}
\end{array}\right) \\
\text { and } \boldsymbol{F}=\left(\begin{array}{lllllll}
F_{0} & F_{1} & F_{2} & F_{3} & F_{4} & F_{5} & F_{6}
\end{array}\right)^{T},(8) \text { is written as: } \\
\boldsymbol{E K}=\boldsymbol{F}
\end{gathered}
$$

According to least square estimation, we can get

$$
\boldsymbol{K}=\left(\boldsymbol{E}^{\boldsymbol{T}} \boldsymbol{E}\right)^{-1} \boldsymbol{E}^{\boldsymbol{T}} \boldsymbol{F}
$$

So we can obtain the distortion parameters using (10).

\section{Algorithm implemention}

Note that we can get the distortion parameters using the proposed algorithm. The algorithm implementation is detailed as follows.

Input: image coordinate $u$ and world screen coordinate $\left[x_{w}, y_{w}\right]$

Output: projective matrix $\mathbf{M}$ and distortion parameters $\boldsymbol{K}$

Step1: variables definition and initial, and compute projective matrix $\mathbf{M}$ initial value

Step2: while $\mathrm{i}<$ itermax $\& \&$ mean error $\eta>$ minimum error expectation \&\& standard variation $\sigma>$ minimum error, goto step3-7.

Step3: solve distortion parameters $\boldsymbol{K}$

Step4: undistortion $u^{\prime}=\mathbf{K} u$

Step5: parameters optimize $(\mathbf{M}$ and $\boldsymbol{K})$

Step6: solve projective matrix $\mathbf{M}$ using undistortion image coordinate $u^{\prime}$

Step7: compute world screen coordinate $\left[x_{w}, y_{w}\right]$ and mean error $\eta$ and standard variation $\sigma$ goto step2

Step8: output error results $\eta, \sigma$ and undistortion coordinate $u^{\prime}$, iterative stop

\section{EXPERIMENTAL RESULTS}

We tested our algorithm on real image using 1D infrared camera, and the calibration points was set by manual which we know theirs ideal world coordinate. To our knowledge, the camera intrinsic parameters are constant, so we assume 
the distortion central is center of the image, and using (11) for error estimation.

$$
\text { error }=|\mathbf{m}(\mathbf{x}, \mathbf{y})-\tilde{\mathbf{M}}(\mathbf{R}, \mathbf{T}, \mathbf{u}, \mathbf{x}, \mathbf{y})|
$$

Where $\mathrm{m}(\mathrm{x}, \mathrm{y})$ is the ideal world coordinate, $\tilde{M}(R, T, u, x, y)$ is obtained by projective matrix. Our results are given in the table below. we can easily see that minimum error is as low as two pixels. Whatever, the mean error in horizon and vertical decreased to thirtieth.

Figure1 gives distortion estimation matches the real distortion distribution. Abscissa denotes camera image coordinate and degrees of distortion is expressed on vertical. Blue cross represents the real distortion, red dot denotes imitated from undistortion. The results show that the method in this paper is good at estimating the distortion parameters.

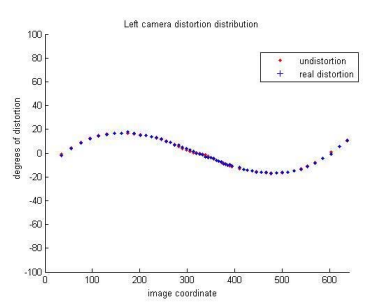

(a) Left camera distortion distribution

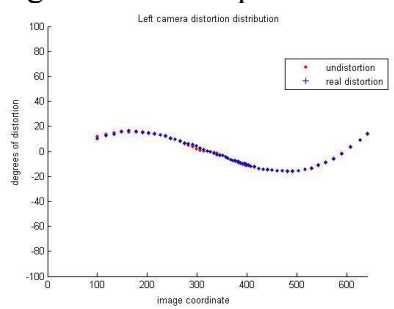

(b) Right camera distortion distribution
Figure 1. Distributions of left and right camera distortion.

We have compared the performance of the method with respect to undistortion and ideal coordinate. As can be seen from Figure2, the undistortion applying our proposed method is close to ideal location. Blue circle represented the ideal coordinate (here get it from projective map), and green cross denoted coordinate before distorting, and red dot was undistortion coordinate.

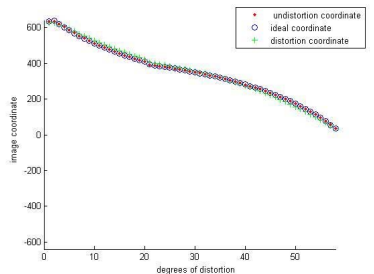

(a) Left: left camera

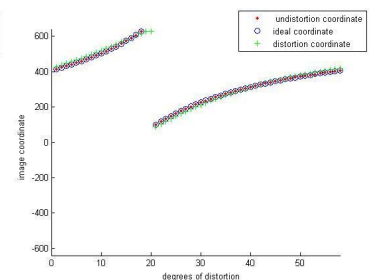

(b) Right: right camera
Figure 2. Results of left and right camera undistorted.

Because there was some image points that were overflowed the range of screen. In order to improve distortion accuracy, we set a bond for screen coordinate in combination with Levenberg-Marquardt [13] optimal algorithm, which did a great help for eliminating outliers (out of screen area). We also employed RANSAC [12] to suppress image noise. Figure 3 depicted the algorithm has a good convergence, which can converge in ten times.

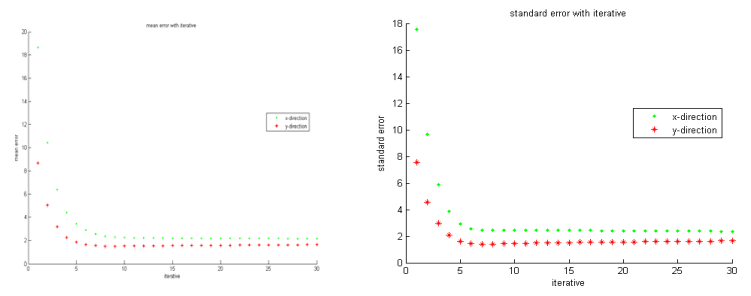

(a)Left: mean error with iterative (b) Right: standard error with iterative

Figure 3. Results of algorithm convergence.

\section{CONCLUSIONS}

In this paper, we show that the estimating of $1 \mathrm{D}$ infrared camera radial distortion can be effectively decoupled from the estimating of other intrinsic and extrinsic camera parameters. Moreover, distortion parameters were determined by linear solution, which not only can reduce computation complex, but also yield accuracy calibration parameteres. In additional, the obtained estimation is accurate enough for many practical applications.

We tested the reliability of our method against noise and outliers(out of screen area). Experiments with real data showed that the technique developed is numerically accuracy with low computation burden, which get high accuracy calibration parameters, even in severe distortion. However, by some effort our method could be extended to some transcendental models. This will be our future work. So we still need a better way to validate the distortion models (e. g, measure the re-projection error).

\section{ACKNOWLEDGEMENT}

This work was supported by Natural Science Foundation of Guangdong Province, China.

\section{REFERENCES}

[1] S. G. Demos, R. Bold, R. V.White, and R. Ramsamooj, Investigationof near-infrared autofluorescence imaging for the detection of breastcancer. IEEE J. Sel. Top. Quantum Electron[J], 2005,11( 4):791-798.

[2] S. G. Demos, R. Bold, R. V.White, and R. Ramsamooj, Investigationof near-infrared autofluorescence imaging for the detection of breastcancer. IEEE J. Sel. Top. Quantum Electron[J], 2005, 11( 4):791-798.

[3] M. Tapia, P. Persi, J. Bohigas, and M. Ferrari-Toniolo, IRASsourcesassociated with small nebulae in star forming regions: Optical and nearinfrared images,Astron. J.[J], 1997, 113: 1769-1787.

[4] L. Colace, G. Masini, F. Galluzzi, and G. Assanto, Evaporated polycrystalline germanium for near infrared photodetection,Microcryst.Nanocryst. Semicond. Mater. Res. Soc. Ed.[J], ,1998, 536: 469-475.

[5] Z. Guoqing, et al., Distortion model selecting and accuracy evaluation for CCD camera calibration, in Signal Processing, 1996, 3rd International

[6] Conference on[C], 1996, 2: 875-878.

[7] Hartley R, Zisserman A, tiple View Geometry in Computer Vision[D]. Camridge :Cambridge University, 2003

[8] A.W. Fitzgibbon. Simultaneous linear estimation of multiple view geometry and lens distortion[A].//In: Proceedings of the IEEE Computer Society Conference onComputer Vision and Pattern Recognition[C], Kauai, HI, USA,2001:125-132 
[9] F. Devernay and O. Faugeras. Straight lines have to be straight. Machine Vision and Applications[J], 2001,13(1):14-24,

[10] R. I. Hartley and T. Saxena. The cubic rational polynomial camera model. In Proc. DARPA Image Understanding Workshop[A], New Orleans, LA,USA, 1997: 649-653.

[11] I. Miyagawa, et al., Simple Camera Calibration From a Single Image Using Five Points on Two Orthogonal 1-D Objects,Image Processing, IEEE Transactions on[J], 2010, 19: 1528-1538,

[12] C. Ricolfe-Viala and A. J. Sanchez-Salmeron, Robust metric calibration of non-linear camera lens distortion. Pattern Recognition[J], 2010,43: 1688-1699.

[13] ZHANG Z. A Flexible new technique for camra calibration,IEEE Transactions on Pattern Analysis and Machine Intelligence.[J], 2000, 22(11): 1330-1334.
[14] M. A. Fischler, R. C. Bolles. Random Sample Consensus: A Paradigm for Model Fitting with Applications to Image Analysis and Automated Cartography. Comm. of the ACM[J], 1981, 24: 381-395.

[15] J. J.Mor' e, The Levenberg-Marquardt algorithm: Implementation and theory, in Numerical Analysis, G. A. Watson, ed., Lecture Notes in Mathematics[J], Springer-Verlag, Berlin, 1978, 630: 105-116.

[16] D. Claus and A. W. Fitzgibbon, A rational function lens distortion model for general cameras. 2005 IEEE Computer Society Conference on Computer Vision and Pattern Recognition, 2005.1: 213-21

TABLE I. RESULTS OF ERROR ANALYSIS

\begin{tabular}{ccccc}
\hline \multirow{2}{*}{ Error type } & Direction & Before distorted (pixel) & After distorted (pixel) & $\begin{array}{c}\text { Projective matrix and distortion parameters } \\
\text { combo optimized (pixel) }\end{array}$ \\
\hline \multirow{2}{*}{ Mean error } & x-direction & 33.3297 & 18.6689 & 2.2617 \\
& y-direction & 17.482 & 8.6578 & 1.516 \\
\hline \multirow{2}{*}{ Standard error } & x-direction & 28.0582 & 17.5571 & 2.4293 \\
& y-direction & 15.353 & 7.5611 & 1.4512 \\
\hline \multirow{2}{*}{ Maximum error } & x-direction & 88.5868 & 58.8143 & 31.8729 \\
& y-direction & 46.5253 & 6.5101 \\
\hline
\end{tabular}

